Z. klin. Chem. u. klin. Biochem.

7. Jg., S. $440-443$, September 1969

\title{
Bestimmung des luteinisierenden Hormons (LH) mit Hilfe eines neuen immunologischen Tests $\left.{ }^{1}\right)$
}

\author{
Von H. ERB und R. H. H. Richter \\ Aus der Universitäts-Frauenklinile Bern (Direktor: Prof. Dr. M. Berger)
}

(Eingegangen am 27. Mai 1969)

\begin{abstract}
Die Technik des neuen immunologischen LH-Tests Luteonosticon wird beschrieben und einer kritischen Betrachtung unterzogen. Insgesamt wurden 394 Urine von insgesamt 33 Frauen mit dem neuen Test untersucht. Insbesondere wurde die Bewertung der Sedimentationsbilder und der Reaktionsmuster diskutiert. Trotz der Störfaktoren und gewisser Unzulänglichkeiten muß dieser semiquantitative Test als nützlich für die Diagnostik in der gynäkologischen und andrologischen Endokrinologie betrachtet werden. Die mit diesem Test durchgeführten Untersuchungen an 14 Patientinnen, denen beide Ovarien chirurgisch entfernt worden waren, zeigten, daß die LHAusscheidung nach der Operation rasch ein Maximum erreicht, anschließend aber wiederum in signifikanter Weise abfällt. Die Ursachen dieser postoperativen Veränderungen der renalen LH-Ausscheidung wurden diskutiert.
\end{abstract}

\section{Determination of luteinising hormone ( $L H)$ with the aid of a nen immunological test}

The operating procedure of the new immunological LH-test Luteonosticon is described and critically assessed. 394 Samples of urine from 33 women were submitted to the new test. In particular, the evaluation of the sedimentation and of the reaction pattern is discussed. In spite of interfering factors and certain inadequacies, this semi-quantitative test is useful for diagnosis in gynaecological and andrological endocrinology. The test was performed on 14 patients, who had both ovaries surgically removed; after the operation the LHexcretion rapidly reached a maximum, then finally showed a significant fall. The reasons for these post operative changes in the renal LHexcretion are discussed.

In der gynäkologischen und andrologischen Endokrinologie ist die Bestimmung des luteinisierenden Hormons LH im Urin sowohl zu diagnostischen als auch wissenschaftlichen Zwecken wertvoll. $\mathrm{Da}$ die biologische Bestimmung schwierig durchzuführen ist, wurde die immunochemische oder immunologische Analyse versucht, wie dies bereits für andere Proteinhormone gemacht worden war. Indessen gestattete bisher nur die radioimmunologische Methode eine direkte Bestimmung im Nativurin, während unter Verwendung des Pregnosticon-Tests der Urin 50-100 fach konzentriert werden mußte. Deshalb und aus andern Gründen wurde die Methode vielfach kritisiert.

Aus diesem Grunde hat die Firma Organon einen neuen Test entwickelt, mit welchem das LH direkt in Nativurin ohne vorherige Konzentration bestimmt werden kann $(1,2)$ und welcher noch in diesem Jahre unter dem Namen Luteonosticon kommerzialisiert werden soll. Der Test beruht auf dem gleichen Prinzip wie der bekannte Schwangerschaftstest Pregnosticon derselben Firma, nämlich auf der Hemmung einer Hämagglutination zwischen Hammelerythrocyten einerseits, welche mit menschlichem Choriongonadotropin (HCG) als Antigen sensibilisiert sind, und Antiserum andererseits, welches durch Immunisierung von Kaninchen mit diesem HCG gewonnen wird. Dabei konnte dieses HCG an Stelle von LH verwendet werden, da zwischen diesen beiden Hormonen eine immunologische Kreuz-Reaktion besteht.

1) Teilweise vorgetragen auf der 13. Generalversammlung der Schweizerischen Vereinigung für Klinische Chemie, 18.-20. April 1969 in Lugano.

\section{Methodik}

Bringt man HCG- (oder IH-) Antiserum mit HCG (oder LH), welches auf Erythrocyten adsorbiert ist, zusammen, so kommt es zur Agglutination der Erythrocyten. Wird diese Agglutination in einem Röhrchen durchgeführt, so wird sie dadurch als solche erkennbar, daß die Erythrocyten nicht mehr normal, sondern zu einem diffusen Bodensatz sedimentieren: das Testresultat ist negativ. Wird nun jedoch mit dem zu untersuchenden Urin eine genügende Menge freies HCG (oder LH) hinzugegeben, so wird das Antiserum dadurch neutralisiert, und die eben beschriebene Agglutination zwischen Antiserum und dem Antigen auf den Erythrocyten kann nicht mehr stattfinden. Somit wird also die Hämagglutination gehemmt, d. b. die Erythrocyten werden nicht agglutiniert, sedimentieren in einem Röhrchen normal und bilden am Boden einen rotbraunen, charakteristischen Ring, welcher durch Spiegelung von unten deutlich zu erkennen ist (s. Abb. 1). Dieser positive Ausfall, diese Hemmung der Hämag-
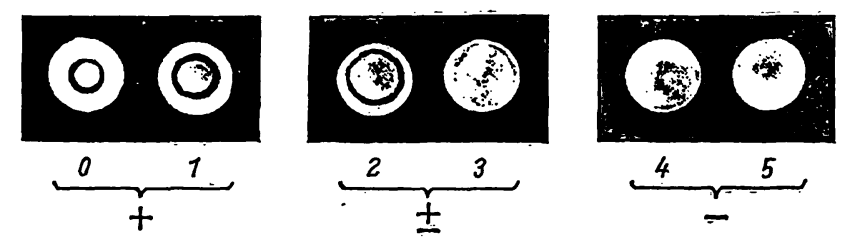

Abb. 1

Sedimentationsbilder

Die Zahlen kennzeichnen den Agglutinationsgrad

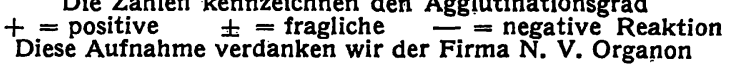

glutination, kann nur dann stattfinden, wenn eine bestimmte Mindestkonzentration von HCG (bzw. LH), welche der Empfindlichkeitsgrenze des Tests entspricht, in dem zu testenden Urin vorhanden ist.

Die Kombinationspackung des neuen LH-Tests Luteonosticon enthält die folgenden drei Reagenzien:

1. das Antiserum von Kaninchen, die mit stark gexeinigtem HCG immunisiert wurden; 


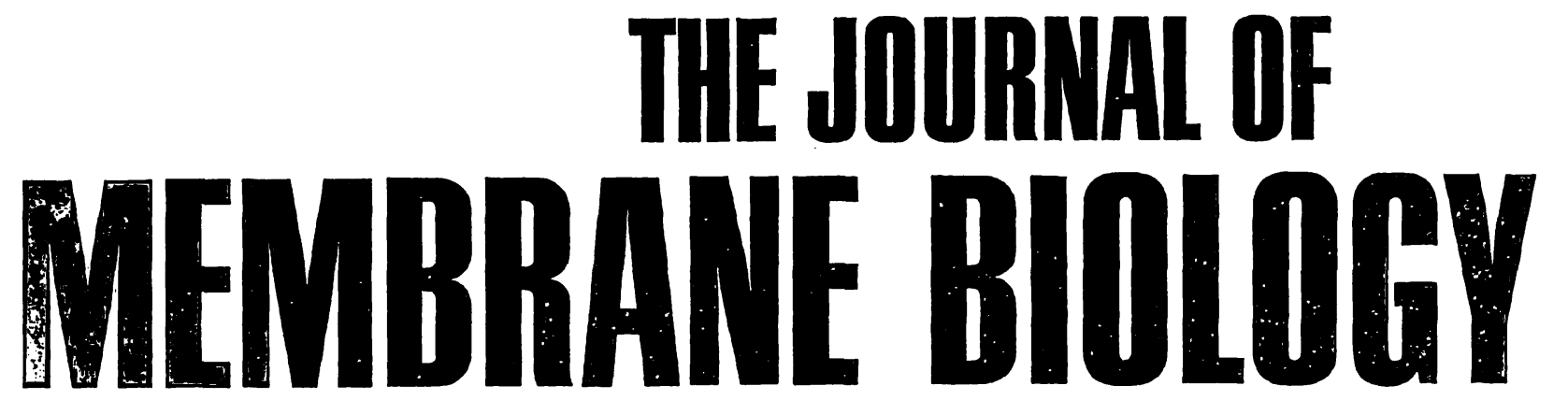

An international journal for studies on the structure, function and genesis of biomembranes

Editorlal Board

W. R. Loewenstein Columbia Editor-in-Chief

P. F. Baker Cambridge

J. M. Diamond California L.A.

I. S. Edelman California S.F.

G. Eisenman Chicago

D. A. Haydon Cambridge

A. Katchalsky Weizmann

M. Kates Ottawa

A. Klug Cambridge

J. H. Law Chicago

A. Leaf Harvard

A. L. Lehninger Johns Hopkins

W. J. Lennarz Johns Hopkins

A. A. Lev USSR Acad. Sc. Leningrad

S. E. Luria MIT

V. Luzzati CNRS, Gif-sur-Yvette

E. A. C. MacRobbie Cambridge

K. J. Mysels Reynolds Co.

D. Noble Oxford

G. E. Palade Rockefeller

H. Passow Saarland, Homburg

B. C. Pressman Pennsylvania

E. Racker Cornell

J. D. Robertson Duke

S. Roseman Johns Hopkins

L. I. Rothfield Connecticut

A. Rothstein Rochester

R. Schlögl MPI Frankfurt

M. M. Shemyakin

USSR Acad. Sc. Moscow

S. J. Singer California, La Jolla

T. Teorell Uppsala

T. E. Thompson Virginia

D. C. Tosteson Duke

H. H. Ussing Copenhagen

W. Wilbrandt Bern

To be published in 1969

Volume 1 (4 issues)

DM 144,-; US $\$ 36.00$

Prospectus on request
The Journal of Membrane Biology will publish papers on the nature, the structure, genesis and functions of biological membranes, and on the physics and chemistry of artificial membranes with a bearing on biomembranes. It will publish articles dealing with plasma membranes (including cell surfaces and accessory surface structures, such as cell walls); intracellular membranes (e. g., membranes of organelles, nuclei, mitochondria, vesicles, intracellular reticula); membrane organs (e. g., skin, urinary bladder, gut, kidney, lung); biomembrane models (e.g., monolayers, bilayers, synthetic membranes); and general considerations of two-dimensional biological structures. Suituable topics are chemical and physical structure; immunochemical properties and fingerprinting; colloid and surface chemistry; transport and secretory functions, including natural and artificial transport carrier systems, diffusion and pinocytosis; metabolic functions, membranebound enzyme systems, transduction; electrical phenomena in excitable membranes, nerve, muscle, receptors, etc.; membrane synthesis and replication; cell regulatory functions of membranes and cybernetic aspects; membrane genetics; evolution and comparative aspects of biomembranes.

Papers will be published in English within 4-6 months after acceptance. The Journal will not publish preliminary notes. It will publish brief letters to the Editor commenting on papers in the Journal. The Journal will also publish occasional review articles. The Editor should be consulted before such a review is submitted.

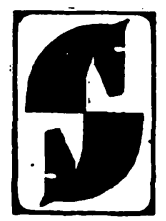
Springer-Verlan New York Inc. 175 Fifth Avenue New York, N. Y. 10010 


\section{Aminosäure- Trennungen können Sie mit dieser Apparatur in 15 Minuten ausführen}

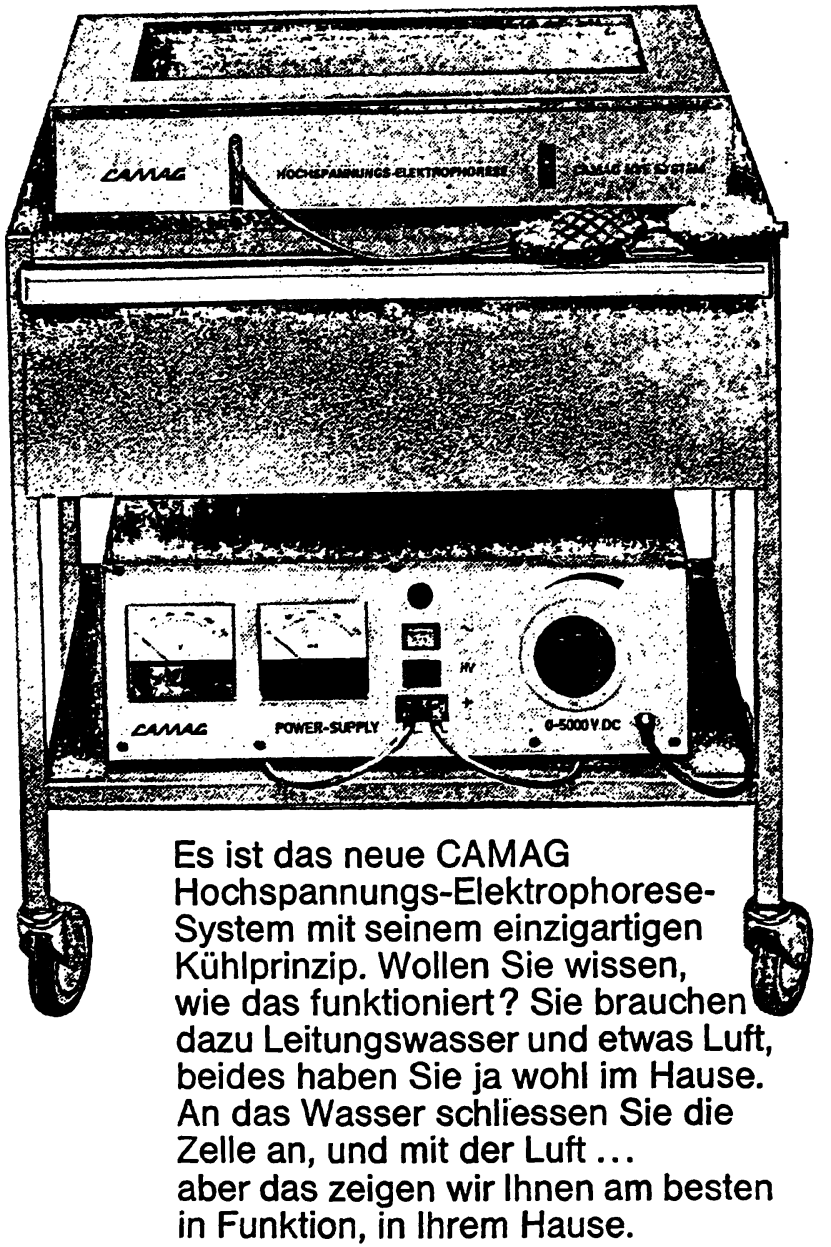

Wenn Sie wollen, schicken wir Ihnen unseren Prospekt, aber lieber kommen wir zu Ihnen mit der ganzen Ausrüstung.

Natürlich können Sie auch andere niedermolekulare Substanzen trennen, z.B.:

Indole, Porphyrine, Zucker, Purine, Vitamine, anorganische lonen.

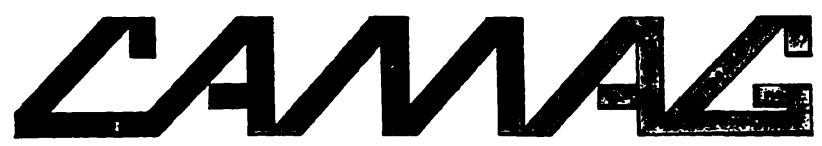

Chemie-Erzeugnisse und Adsorptionstechnik AG Homburgerstrasse 244132 Muttenz/Schweiz

Unser Zweigbetrieb in der Bundesrepublik: CAMAG, 1000 Berlin 45, Baseler Strasse 65
ARBEITSMETHODEN

DER MODERNEN NATURWISSENSCHAFTE

Herausgegeben von Prof. Dr. Kurt Fischbeck

\section{Pataki}

\section{Dünnschichtchromatographie}

in der Aminosäure- und Peptidchemie

Von Dr. phil., Ing. Chem. György Pataki

Oktav. Mit 128 Abbildungen, 52 Tabellen,

1 Ausschlagtafel, 463 Literaturangaben.

XX, 250 Seiten. 1966. Plastikeinband DM 38,-

Das Buch ist empfehlenswert: es stellt für jeden, der al diesem Gebiet arbeitet, eine wertvolle Hilfe und ein guten Ratgeber für die Praxis dar. Die Erfahrung dı Verfassers bewirkt eine solide Auswahl und klare Inte. pretation der Methoden wie deren Möglichkeiten.

Zeitsclurift für Kliniscbe Cbem

\section{Maurer \\ Disk-Elektrophorese}

Theorie und Praxis der diskontinuicrlichen

Polyacrylamdidgel-Elektrophorese

von Dr. rer. nat. H. RAINER MAURER

Mit einem Geleitwort von E. HecKer

Oktav. XVI, 221 Seiten. Mit 82 Abbildungen, 15 Tabellen, 1 Ausschlagtafel und 578 Literaturangaben 1968. Plastikeinband DM 36,-

Die Monographie stellt eine abgerundete Übersicht über das gesamte Gebiet der Disk-Elektrophorese dar. Das Erscheinen dieses Werkes ist sehr zu begrüßen, da die Trennungen von Proteinen und anderen diversen Polymeren mit der Disk-Elektrophorese eine wachsende Bedeutung in den verschiedensten Fachgebieten erlangen.

Die theoretischen Grundlagen sind kurz, einfach und präzise dargestellt. Daneben wird auf umfangreichere Abhandlungen verwiesen.

Der praktische Teil des Buches steht im Vordergrund. Es werden die Methodik der analytischen Disk-Elektrophorese und die präparative Polyacrylamidgel-Elektrophorese abgehandelt. Besonders hervorzuheben sind die straffe Gliederung, die gut gestalteten Abbildungen und Tabellen, die verständlichen Arbeitsvorschriften, die Einschätzung der Vor- und Nachteile der einzelnen Verfahren und die Hinweise auf Fehlermöglichkeiten.

Das Ärztliche Laboratorium, Berlin

Walter de Gruyter \& Co $\cdot$ Berlin 
2. Erythrocyten, die mit HCG sensibilisiert worden sind;

3. Verdünnungsflüssigkeit zum Resuspendieren der Erythrocyten, Lösen des lyophilisierten Antiserums, zum Verdünnen des Urins und für die Kontrollreaktionen.

Ein wesentlicher Unterschied von Luteonosticon zu Pregnosticon ist seine stark gesteigerte Empfindlichkeit; denn Luteonosticon gibt bereits beim Vorhandensein von 15 i. E. LH pro Liter eine positive Reaktion.

Um zu einer quantitativen Aussage zu kommen, muß mit einer Verdünnungsreihe des zu testenden Urins gearbeitet werden. Normalerweise werden fünf Konzentrationen angesetzt und das sechste Röhrchen dient als positive Blindprobe (Abb. 2). Sollte

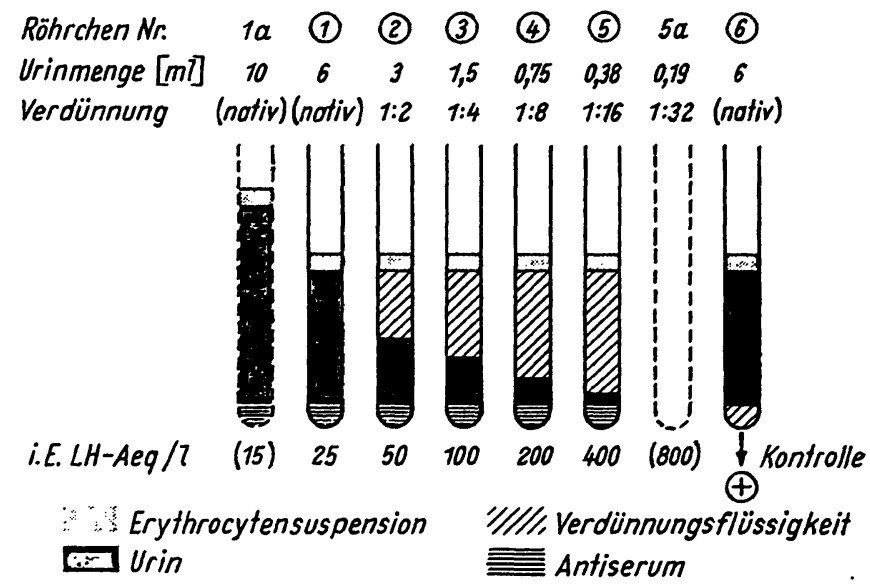

Abb. 2

Aufstellen der Verdünnungsreihe

auch in der größten Verdünnung, im Röhrchen Nr. 5, noch eine positive Reaktion auftreten, so kann man weitere Verdünnungen testen. Tritt andererseits im Nativurin, Röhrchen Nr. 1, noch eine negative Reaktion ein, so kann durch Vermehrung des Urinvolumens der Test noch etwas empfindlicher gestaltet werden. In die Röhrchen 1 bis 5 gibt man zunächst das Antiserum, in die Röhrchen 2 bis 5 steigende Mengen eines Puffers als Verdünnungsflüssigkeit, hierauf den Urin in Röhrchen 1 bis 6 , in Röhrchen 1 bis 5 in absteigenden Mengen $(6 ; 3 ; 1,5 ; 0,75$ und $0,38 \mathrm{~m} /$ ). In Röhrchen Nr. 6 wird das Antiserum durch den Puffer ersetzt. Die Reagenzien werden gemischt, und man läßt 2-4 Stdn. stehen. Hierauf gibt man die Erythrocyten-Suspension hinzu, mischt und läßt erneut 30-60 Min. stehen. Sodann werden die Röhrchen zentrifugiert, das Uberstehende wird dekantiert, die Erythrocyten werden ausgewaschen und hierauf resuspendiert. Nach 2 Stdn. kann man die Reaktion ablesen. Dabei sind nicht nur positive und negative Reaktionen zu erkennen, sondern auch sogenannte zweifelhafte, bei denen der rotbraune Ring entwedex zu groß, zu dünn odex defekt erscheint (Abb. 1). Alle Urinproben wurden eingefroren, bei $-15^{\circ}$ aufbewahrt und erst kurz vor der Analyse aufgetaut.

\section{Bewertung}

Zur Bewertung der Resultate wird folgendermaßen vorgegangen: Eine positive Reaktion im Röhrchen Nr. 1 (Abb. 2) bedeutet, daß pro Liter Urin mindestens 25 i. E. LH immunochemisch bestimmt bzw. LH-Aeq. vorhanden sind. Eine positive Reaktion im Röhrchen Nr. 2, aber eine negative oder zweifelhafte im Röhrchen Nr. 3 bedeutet, daß 50 i. E. LH-Aeq./l Urin vorhanden sind usw. Die Blindprobe im Röhrchen Nr. 6 muß natürlich stets eine positive Reaktion ergeben.

Auf Grund der Vorschläge der Herstellerfirma kann zwischen positivem, intermediärem (,zweifelhaftem ") und negativem Ausfall der Reaktion, bzw. zwischen to- tal sechs verschiedenen Sedimentationsbildern (Abb.1) unterschieden werden. Die Stadien 0 und 1 gelten als positiv ( + , die Stadien 2 und 3 als intermediär bzw. zweifelhaft $( \pm)$ und die Stadien 4 und 5 als negativ $(-)$. Die intermediären Sedimentationsbilder beruhen auf einer unvollständigen, bzw. teilweisen Hemmung der Hāmagglutination, wãhrend die positiven einer vollständigen oder nahezu vollständigen Hemmung entsprechen. Wir haben den Eindruck, daß der Test unter Heranziehung der intermediären Reaktionen zur Berechnung der Resultate nicht nur prãziser, sondern auch empfindlicher gestaltet werden kann, worauf auch LASCHET und LASCHET (3) hinweisen. Es wäre wohl von Vorteil, wenn mit jeder Untersuchungsreihe ein für die immunologische Reaktion geeigneter Standard mitgeführt würde, damit die Sedimentationsbilder direkt verglichen werden können. Auch würde dann eine Verdünnungsreihe mit geringeren Stufen (z. B. 1:1,4:2:2,8: 4 usw.) (gegenüber der gegenwärtig routinemäßig verwendeten Verdünnungsreihe von 1:2:4 usw.) sinnvoll sein. Trotzdem verzichteten wir darauf und bezogen uns bei der Berechnung stets nur auf diejenige größte Urinverdünnung, die eben noch einen "deutlich positiven“" Ausfall ergab, d. h. die Sedimentationsbilder 0 oder 1 aufwies. Dazu haben wir uns aus zwei Gründen entschlossen:

1. benötigt die Beurteilung intermediärer Reaktionsbilder mehr Erfahrung als das sichere Erkennen der "deutlich positiven" Reaktion, und

2. entsprach diese Bewertungsweise einem Beschluß, welcher an einem Zusammentreffen aller Schweizer Untersuchergruppen gefaßt worden war. Allerdings ist diese von uns gewãhlte Bewertungsweise nicht völlig befriedigend, sei es aus Gründen, die weiter unten noch dargelegt werden, sei es, weil die Unterscheidung zwischen den Stadien 1 ("noch positiv") und 2 („,bereits zweifelhaft") Schwierigkeiten bereitet.

Es scheint, daB „Störfaktoren“" verschiedener Art den deutlichen Ausfall der Reaktion beeinträchtigen können. Diese führen dazu, daß abnorme Reaktionsmuster (= Reihenfolge der Reaktionen von Röhrchen 1 bis 5) entstehen, wie sie in Tabelle 1 aufgeführt sind. Die Bewertung haben wir in diesen Fällen streng nach dem vorhin beschriebenen Prinzip vorgenommen, d. h. immer wurde diejenige letzte Verdünnung in der Verdünnungsreihe zur Berechnung herangezogen, welche eben noch eine „deutlich positive“ Reaktion ergab. Wir sind uns jedoch bewußt, daß solche Resultate sehr unsicher sind. Fiel die positive Kontrollprobe (Röhrchen Nr. 6) negativ aus, so wurde - entsprechend den Vorschlägen der Herstellerfirma - der Urin während 5 Min. erwärmt (zur Zerstörung gewisser Inhibitoren) und hierauf die Reaktion neu angesetzt. Nur bei drei von neun Urinen blieb die Blindprobe auch nach vorherigem Erwärmen des Urins negativ; alle drei Urine stammten jedoch von der gleichen Patientin. In diesen Fällen wurde auf eine Bewertung verzichtet.

Wenn die "deutlich positive" Reaktion (Sedimentationsbild 0 oder 1 ) innerhalb der Verdünnungsreihe ab- 
Tab. 1

Abnorme Reaktionsmuster, ihre Häufigkeit und Bewertung

\begin{tabular}{|c|c|c|c|c|c|c|c|c|c|}
\hline \multirow[b]{2}{*}{ Röhrchen Nr. } & \multicolumn{5}{|c|}{$\begin{array}{l}\text { Reaktionsmuster (Sedimentationsbilder } \\
\text { im Röhrchen } 1-5 \text { ) }\end{array}$} & \multirow{2}{*}{$\begin{array}{c}\text { Reaktionsmuster } \\
\text { der } \\
\text { Kontrollprobe } \\
6\end{array}$} & \multirow[t]{2}{*}{ Typ } & \multirow{2}{*}{$\begin{array}{c}\text { Bewertung } \\
\text { in i. E. } \\
\text { LH immund- } \\
\text { chem. bestimmt }\end{array}$} & \multirow{2}{*}{$\begin{array}{l}\text { Häufigkeit } \\
(\%)\end{array}$} \\
\hline & 1 & 2 & 3 & 4 & 5 & & & & \\
\hline \multirow[t]{10}{*}{ Verdünnung } & nativ & $1: 2$ & $1: 4$ & $1: 8$ & $1: 16$ & nativ & & & \\
\hline & + & + & \pm & + & - & + & $\mathbf{A}$ & 200 & 1,5 \\
\hline & + & + & \pm & \pm & - & + & B & 50 & 11,7 \\
\hline & + & + & \pm & \pm & \pm & + & C & 50 & 1,3 \\
\hline & \pm & $\doteq$ & \pm & 二 & 二 & + & D & $<25$ & 1,5 \\
\hline & \pm & 二 & 二 & - & - & + & $\mathrm{E}$ & $<25$ & 3,0 \\
\hline & 二 & + & + & + & - & - & $\mathbf{F}$ & - & 0,8 \\
\hline & - & - & - & - & - & - & $\mathbf{G}$ & - & 1,5 \\
\hline & - & + & + & + & - & + & $\mathbf{H}$ & 200 & 1,0 \\
\hline & \pm & + & \pm & \pm & - & + & $I$ & 50 & 0,5 \\
\hline
\end{tabular}

A Eine Reihe positiver Reaktionen unterbrochen von einem oder zwei intermediären oder negativen Reaktionen (Bilder 2, 3, 4 oder 5 )

B Ein oder mehr positive Reaktionen gefolgt von zwei intermediären (Bilder 2 oder 3 )

D In zwei oder mehr Röhrchen intermediäre Reaktionen, keine positiven

E Nur im Röhrchen Nr. 1 eine intermediäre Reaktion, in allen andern negative Reaktionen

$F$ Eine oder mehrere negative Reaktionen, gefolgt von positiven; Kontrollprobe negativ!

$G$ Alle Reaktionen negativ; Kontrollprobe negativ!

H Eine oder mehrere negative Reaktionen, gefolgt von positiven, Kontrollprobe aber positiv

I Eine intermediäre Reaktion im Röhrchen Nr. 1, gefolgt von einer positiven Reaktion; Kontrollprobe positiv.

nimmt, so sollten theoretisch wohl die sich nachfolgenden Sedimentationsbilder von einem Röhrchen zum andern sich jeweils um mindestens zwei Stufen voneinander unterscheiden, d. h. die Muster sollten z. B. folgender-

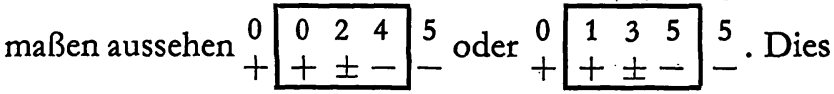
ist aber leider oft nicht der Fall (siehe Tab. 1), so daß Unsicherheiten in der Bewertung entstehen. Bei Mustern, bei welchen ein Reaktionsbild 2 auftritt, ist stets damit zu rechnen, daß es sich um Stufe 1 gehandelt hat; es ist deshalb dann mit der Möglichkeit eines Fehlers von einer ganzen Verdünnungsstufe (Faktor 2) zu rechnen. Das ideale Muster $\begin{array}{r}0 \\ \left.+\begin{array}{rrr}0 & 4 & 5 \\ + & - & -\end{array}\right]\end{array}$ gang von einer völlig positiven zu einer negativen Reaktion unter Umgehung der intermediären Reaktionsstufen kommt leider nicht häufig vor. Unter Berücksichtigung der jetzigen Bewertung und auf Grund unserer Erfahrungen glauben wir mit einer Präzision, bzw. Reproduzierbarkeit rechnen zu dürfen, die für einen semiquantitativen Test befriedigend ist; wir würden jedoch annehmen, daß zwei einzelne Resultate, die sich lediglich durch den Faktor 2 unterscheiden, nicht signifikant verschieden sind. Es ist demnach empfehlenswert, die Bestimmungen doppelt oder gar dreifach durchzuführen.

\section{Resultate}

Insgesamt wurden 394 Urine von total 33 Patientinnen untersucht. $69^{\circ}(=17,5 \%)$ Urine gaben Reaktionsmuster, aus denen nur ein relativ unsicheres Resultat abzulesen war (Typ des Reaktionsmusters A, B, C, D, $H$ und $I$ in Tab. 1). Insgesamt wiesen $72(=18,3 \%)$ Urinproben mindestens ein Sedimentationsbild 2 (ohne Bild 1) auf, ein Bild also, welches als intermediär zu bezeichnen ist und infolgedessen nicht zur Berechnung des Resultates miteinbezogen wurde. Dieses Bild 2 hätte aber unseres Erachtens in den meisten Fällen auch als ein Bild 1 abgelesen werden können, besonders wenn kein Vergleich mit einem Bild 1 gleichzeitig möglich war. Dadurch hätte sich das Resultat aber um Faktor 2 vergrößert. Andererseits ergaben $88(=22,3 \%)$ Urine als letzte "deutlich positive" Reaktion ein Sedimentationsbild 1 (gefolgt von 3, 4 oder 5), welches auch als 2 hätte gedeutet werden können, wodurch das Resultat sich auf die Hälfte verringert hätte. Nur in $101(=25,6 \%)$ von 394 Fällen traten keine intermediären Sedimentationsbilder auf, d. h. war ein direkter Úbergang von positiv ( 0 oder 1 ) nach negativ (4 oder 5) vorhanden. In unserem Material waren bei $18(=4,6 \%)$ Urinproben alle Reaktionen negativ, d. h. sie enthielten also < 25 i. E. LH-Aeq. $/ /$; bei $39(=9,9 \%)$ Ürinproben waren alle Reaktionen positiv, d. h. diese enthielten mindestens 400 i. E. LH-Aeq./l. Die Nachprüfung in diesen Fällen auf 800 i. E. LH-Aeq./l ergab in 34 Fällen ein negatives, in 5 Fällen ein positives Resultat.

Trotz der verschiedenen Schwierigkeiten erscheinen uns die von uns erhaltenen Resultate sinnvoll. Sie wurden sowohl pro Liter oder pro 24 Stunden als auch auf $1,5 \mathrm{~g}$ Kreatinin - entsprechend den Vorschlägen der Herstellerfirma - berechnet. Unsere Werte liegen zwischen $<25$ i. E. LH-Aeq. $/ l$ bzw. $<14$ i. E. LHAeq./1,5 g Kreatinin bzw. < 16 i. E. LH-Aeq./24Stdn. und 800 i. E. LH-Aeq. $/ l$ bzw. 1582 i. E. LHH-Âeq. $/ 1,5 \mathrm{~g}$ Kreatinin bzw. 920 i. E. LH-Aeq./24 Stdn.

Wir hatten uns die Aufgabe gestellt, mit Hilfe von Luteonosticon abzuklären, welchen Einfluß die Entfernung beider Ovarien, also die chirurgische Kastration der Frau, auf die renale Ausscheidung des luteinisierenden Hormons hat. Hierzu wurde bei 14 Frauen (total 210 Urinproben), denen beide Ovarien operativ entfernt wurden, entsprechende Untersuchungen durchgeführt, und zwat vor der Operation während 1 bis 4 Tagen, postoperativ während 11 bis 13 Tagen. Präoperativ wurden mehrheitlich Morgenurine, am Opera- 
tionstag selbst und an den 2 bis 5 folgenden Tagen 24-Stunden-Urine gesammelt und untersucht, ebenso jeweils am letzten Tag einer Untersuchungsreihe. An den Zwischentagen mußten wir uns auf die Untersuchung des Morgenurins beschränken.

Die für die einzelnen Urine gefundenen Werte, die statistische Bearbeitung und die daraus hervorgehenden Resultate werden in extenso in 1. c. (4) publiziert. Wegen der großen Variation zwischen den Werten der verschiedenen Patientinnen wurden die absoluten Werte zunächst „standardisiert", d. h. sie wurden durch die entsprechenden Patientendurchschnittwerte dividiert und hierauf logarithmisch aufgetragen. Die arithmetischen Mittelwerte der einzelnen Tage ergaben die in Abbildung 3 wiedergegebene Kurve. Der Mittelwert

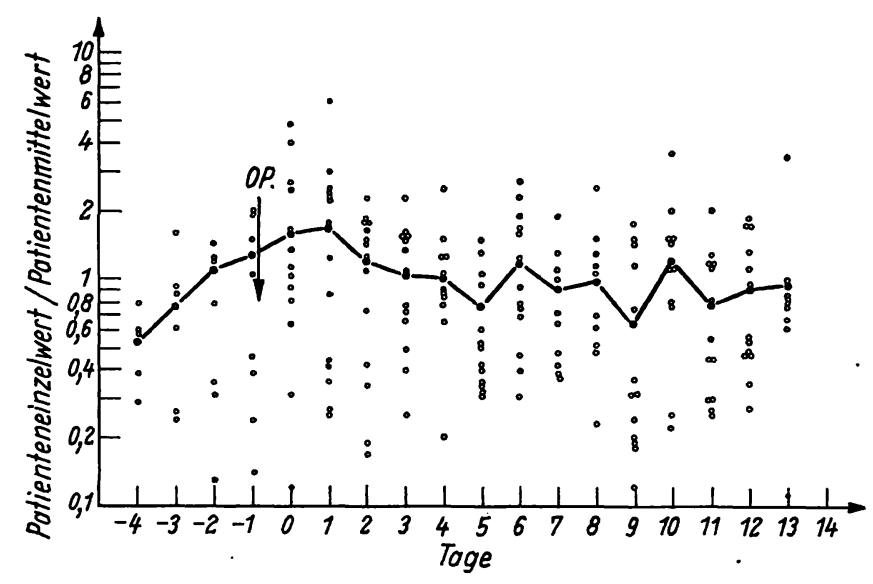

Abb. 3

LH-Tagesausscheidung vor und nach Ovarektomie. Standardisierte kumulative Kurve. Alle einzelnen Patientenwerte (i. E. LH.-Aeq. immunochemisch bestimmt $/ 1,5 \mathrm{~g}$ Kreatinin) wurden durch die jeweiligen Patientendurchschnittswerte dividiert und als Quotienten auf die Ordinate aufgetragen. Die ausgezogene Linie verbindet die arithTage. Der Operationstag wurde mit 0 bezeichnet, die pröoperativen Tage mit negativen Ziffern, die postoperativen Tage mit positiven

der Resultate der präoperativen Tage -4 bis -1 unterscheidet sich in signifikanter Weise vom Mittelwert der Resultate der postoperativen Tage 0 bis $+3(P=0,05)$. Der Kurvenabfall vom Tag 1 bis Tag 9 ist hoch signifikant $(P<0,001)$. Auf Grund dieser Tatsachen scheint uns ein postoperatives Maximum und ein darauffolgender Abfall der LH-Ausscheidung nach beidseitiger Ovarektomie hinreichend bewiesen zu sein. Der auf der Kurve sichtbare „Anstieg“ der Ausscheidung vom Tag
-4 bis zum Tag +1 ist rein zufällig; die Berechnung der Regression ergab keine Signifikanz $(P=0,76)$.

Die Variation der Kreatininausscheidung von Tag zu Tag ist bei den einzelnen Patientinnen nur unbedeutend bzw. nicht so groß, daß sie unsere Befunde wesentlich hätte beeinflussen können (4).

Es darf wohl angenommen werden, daß die schlagartige Ausschaltung der endokrinen Ovarialfunktion eine rasch einsetzende Vermehrung der LH-Ausschüttung aus der Hypophyse zur Folge hat. Der nachfolgende Abfall könnte zwei verschiedenen Einflüssen zugeschrieben werden:

1. wäre es möglich, daß der durch die Kastration bedingte erhöhte LH-Serumspiegel die weitere Produktion bzw. Ausschüttung von LH-RF aus dem Hypothalamus bzw. von $\mathrm{LH}$ aus der Hypophyse hemmt; für die Existenz eines solchen negativen Rückkupplungsmechanismus für LH konnten von KaWAKAMI und SAWYER (5), Ramirez und Sawyer (6), Corbin und Cohen $(7,8)$ sowie von David, Fraschini und Martini (9) Beweise erbracht werden.

2. könnte aber der Abfall der IH-Ausscheidung auch durch den operativen Streß bedingt sein; kürzlich haben ODELL und Mitarbeiter (10) einen solchen Effekt mit Hilfe der radioimmunologischen Methode nachweisen können.

\section{Schlußfolgerung}

Trotz der. gelegentlich auftretenden Störfaktoren, die sich in einem unscharfen Umschlagspunkt manifestieren und die Beurteilung des Reaktionsausfalls erschweren, trotz der relativ geringen Präzision und trotz der bekannten Tatsache, da $B$ die immunologische Reaktion nicht notwendigerweise mit der biologischen identisch ist, möchten wir meinen, $\mathrm{da} ß \mathrm{der}$ von uns untersuchte LH-Test zu diagnostischen Zwecken durchaus brauchbar ist und manch nützlichen Hinweis geben kann. Weitere Untersuchungen hinsichtlich der Reproduzierbarkeit, der Störfaktoren und der Spezifität sind sicher wünschenswert. Die genannten Mängel sind insbeson'dere in pathologischen Fällen zu berücksichtigen, und man muß sich bewußt sein, wo die Möglichkeiten und Grenzen dieses Tests liegen.

Wir danken Herrn Prof. Dr. S. Rosin für seine wertvollen Ratschläge zur statistischen Bearbeitung und Fräulein Elisabetr ACKermanN für ihre technische Assistenz.

\section{Literatur}

1. Sçruurs, A. H. W. M., Acta endocr. (K'hvn), Suppl. 119, 129 (1967). - 2. SchuURs, A. H. W. M., E. JAGER und J. D. H. Homan, Acta endocr. (K'hvn), 59, 120 (1968). - 3. LASCHET, L. und U. LASCHET, Acta endocr. (K'hvn), Suppl. (im Druck). - 4. ERB, H. und R.H.H. Richrer, Acta endocr. (K'hvn), Suppl. (im Druck). 5. Kawakami, M. und C. H. SAwYER, Endoctinology 65, 631 (1959). - 6. RAMIEEZ, V. D. und C.H. SAwYER, Endocrinology 76, 282 (1965). - 7. Corbin, A. und A. I. Cohen, Endocrinology
78, 41 (1966). - 8. Corbin, A., Endocrinology 78, 893 (1966). 9. David, M. A., F. Fraschini und L. Martini, Endocrinology 78, 55 (1966). - 10. Charters; C., W. D. Odell und J. ThompSON, zitiert von SWERDLOFF, R. S. und W. D. ODELL, in Rosemberg, E., Gonadotropins 1968, Proceedings of the Workshop Conference, Seiten 155-162, Vista Hermosa, Mor., Mexico 1968 (Geron-X, Inc., Los Altos, Calif., USA, 1968).

PD Dr. med. H. Erb und Dr. phil R. H. H. Richter Universitäts-Frauenklinik $\mathrm{CH}-3001$ Bern 